\title{
Bose-Einstein condensation and chiral phase transition in linear sigma model
}

\author{
Song Shu $\dagger$ and Jia-Rong Li \\ Institute of Particle Physics, Hua-Zhong Normal University, Wuhan 430079, P. R. \\ China
}

\begin{abstract}
With the linear sigma model, we have studied Bose-Einstein condensation and the chiral phase transition in the chiral limit for an interacting pion system. A $\mu-T$ phase diagram including these two phenomena is presented. It is found that the phase plane has been divided into three areas: the Bose-Einstein condensation area, the chiral symmetry broken phase area and the chiral symmetry restored phase area. Bose-Einstein condensation can happen either from the chiral symmetry broken phase or from the restored phase. We show that the onset of the chiral phase transition is restricted in the area where there is no Bose-Einstein condensation.
\end{abstract}

PACS numbers: 11.10.Wx, 11.30.Rd, 03.75.Nt 


\section{Introduction}

In relativistic heavy ion collisions, large amount of pions can be created in small spacetime regions. Theoretically, it is possible for these pions to condensate into the zero momentum and form Bose-Einstein condensation(BEC) at certain temperatures and densities. For this problem, there are some discussions in the literature, for example, the BEC of pions in the non-equilibrium process 1, 2, the pion condensation in thermal equilibrium incorporating boundary effects [3], and the search for the "cold spots" in multipion system [4, 5], etc. These studies show a great interest on this issue. On the other hand, in high energy physics, the chiral symmetry which is spontaneously broken at low temperature is expected to be restored at high temperature and/or density [6]. Long before, Kapusta and Haber had discussed the relation between BEC and the spontaneous symmetry breaking by using different scalar models [7, 8]. Their main interest, however, was focused on the physics of the electroweak system. Here in the strong interacting pion system, one may wonder whether there are some relations between the chiral symmetry restoration and BEC.

Our motivation in this paper is to discuss the relation between these two phenomena in the pion system and find the phase diagram of them through a model study. As far as we know, this topic has not been much discussed in the literature. In [9], the chiral symmetry restoration is discussed in the interaction pion gas through virial expansion, where BEC is only discussed in the case of free pion gas. Here we will discuss BEC and the chiral phase transition in the interacting pion system with the linear sigma model. The linear sigma model has been extensively used to discuss the chiral symmetry restoration at finite temperature [10, 11, 12. In our discussion, to address BEC and the chiral phase transition simultaneously, we will consider a finite pion density as well as the temperature. The pion chemical potential can be introduced in different ways. Theoretically, at given conditions when the pion number can be regarded as being fixed [13, one could introduce the pion number density. However, generally speaking, pion number is not a conserved quantity and one should find a certain conserved charge of the system. For the linear sigma model which has the isospin invariance, one can introduce a chemical potential associated with the isospin charge. For example, in [14], the pion isospin chemical potential is introduced by the third component of the isospin charge and applied to the discussions of the thermal pion mass within the chiral perturbation theory. In our discussion, the isospin chemical potential of pions is introduced to the linear sigma model in the same way.

The organization of the present paper is as follows. In section 2 the effective potential for the linear sigma model is calculated within the Cornwall, Jackiw, Tomboulis resummation scheme at finite temperature and finite pion density in the Hartree approximation. The gap equations are derived by the stationary condition, which form the basis of our numerical calculation. In section 3, the BEC and the chiral phase transition are studied by a numerical calculation. We discuss their $\mu-T$ phase diagrams and give the physical analysis. The last section comprises a summary of our results. 


\section{CJT formalism and effective Lagrangian}

In studies of phase transitions the effective potential is an important and popular theoretical tool. It is defined through an effective action which is the generating functional of the one particle irreducible graphs. A generalized version is the effective potential for composite operators introduced by Cornwall, Jackiw and Tomboulis(CJT) [15]. This formalism assumes that the effective potential $V(\phi, G)$ depends not only on $\phi(x)$, a possible expectation value of the quantum field $\Phi(x)$, but also on $G(x, y)$, a possible expectation value of the time-ordered product $T \Phi(x) \Phi(y)$. Physical solutions demand minimization of the effective potential with respect to both $\phi$ and $G$, which means

$$
\frac{d V(\phi, G)}{d \phi}=0, \quad \frac{d V(\phi, G)}{d G}=0
$$

This formalism was originally written at zero temperature. Then it has been extended to finite temperature occasion by Amelino-Camelia and Pi for investigations of the effective potential of the $\lambda \phi^{4}$ theory [16]. It has been also used in the linear sigma model for discussing the chiral phase transition at finite temperature [10]. At finite density as well as finite temperature, we will employ this formalism to discuss both BEC and the chiral phase transition with the linear sigma model.

For the linear sigma model, here we will ignore the fermion sector. The Lagrangian can be written as

$$
\begin{aligned}
\mathcal{L}=\frac{1}{2}(\partial \sigma)^{2} & +\frac{1}{2}(\partial \vec{\pi})^{2}-\frac{1}{2} m^{2} \sigma^{2}-\frac{1}{2} m^{2} \vec{\pi}^{2} \\
& -\frac{\lambda}{24}\left(\sigma^{2}+\vec{\pi}^{2}\right)^{2}-\varepsilon \sigma
\end{aligned}
$$

where $\sigma$ and $\vec{\pi}$ are the sigma field and the three pion fields $\left(\pi_{1}, \pi_{2}, \pi_{3}\right)$ respectively. The explicit chiral symmetry breaking term is $\varepsilon=f_{\pi} m_{\pi}^{2}$, where $f_{\pi}=93 \mathrm{MeV}$ is the pion decay constant. The coupling constant $\lambda$ and negative mass parameter $m^{2}$ of the model are chosen to be $\lambda=3\left(m_{\sigma}^{2}-m_{\pi}^{2}\right) / f_{\pi}^{2}$ and $-m^{2}=\left(m_{\sigma}^{2}-3 m_{\pi}^{2}\right) / 2>0$. In our discussion, we only consider the case in the chiral limit $\varepsilon=0$, then at zero temperature the pion mass is $m_{\pi}=0$ and the sigma mass is taken as $m_{\sigma} \approx 600 \mathrm{MeV}$.

The conventional treatment of spontaneous chiral symmetry breaking of the linear sigma model is to shift the sigma field as $\sigma \rightarrow \sigma+\phi$, where $\phi$ is expectation value of the sigma field and the order parameter of the chiral phase transition. Then the Lagrangian results as

$$
\begin{aligned}
\mathcal{L}=\frac{1}{2}(\partial \vec{\pi})^{2} & +\frac{1}{2}(\partial \sigma)^{2}-\frac{1}{2}\left(m^{2}+\frac{\lambda}{2} \phi^{2}\right) \sigma^{2}-\frac{1}{2}\left(m^{2}+\frac{\lambda}{6} \phi^{2}\right) \vec{\pi}^{2} \\
& -\frac{1}{2} m^{2} \phi^{2}-\frac{\lambda}{24} \phi^{4}-\frac{\lambda}{24}\left(\sigma^{2}+\vec{\pi}^{2}\right)^{2}-\frac{\lambda}{6} \phi \sigma^{3}-\frac{\lambda}{6} \phi \sigma \pi^{2} .
\end{aligned}
$$

In the following discussion, we will adopt Hartree approximation which means only the bubble diagrams with four-point vertex need to be calculated. So the last two terms in the Lagrangian are neglected [10]. 
The chemical potential is introduced through the third component of isospin charge. The Lagrangian (3) is invariant under the isospin transformation, therefore according to Noether's theorem the conserved current is $\vec{V}_{\mu}=\vec{\pi} \times \partial_{\mu} \vec{\pi}$ and the conserved isospin charge is $\vec{V}_{0}=\vec{\pi} \times \partial_{0} \vec{\pi}$. Then the partition function of the system can be written as

$$
Z=\int\left[d \Pi_{\vec{\pi}}\right]\left[d \Pi_{\sigma}\right][d \vec{\pi}][d \sigma] \exp \left[i \int d^{4} x\left(\mathcal{L}+\vec{\mu}_{I} \cdot \vec{V}_{0}\right)\right],
$$

where $\Pi_{\vec{\pi}}$ and $\Pi_{\sigma}$ are the conjugate momenta of $\vec{\pi}$ and $\sigma$. The isospin chemical potential $\vec{\mu}_{I}=(0,0, \mu)$, in which $\mu$ is the third component of isospin chemical potential, is introduced associated with $\vec{V}_{0}[14$, thus

$$
\vec{\mu}_{I} \cdot \vec{V}_{0}=\mu\left(\pi_{1} \partial_{0} \pi_{2}-\pi_{2} \partial_{0} \pi_{1}\right) .
$$

Here it is more convenient to work with fields possessing well-defined charges:

$$
\pi_{-} \equiv \frac{1}{\sqrt{2}}\left(\pi_{1}+i \pi_{2}\right), \quad \pi_{+} \equiv \frac{1}{\sqrt{2}}\left(\pi_{1}-i \pi_{2}\right), \quad \pi_{0} \equiv \pi_{3} .
$$

Integrating out the conjugate momenta in equation (4) and using the redefined pion fields, one obtains

$$
Z=\int\left[d \pi_{+}\right]\left[d \pi_{-}\right]\left[d \pi_{0}\right][d \sigma] \exp \left[i \int d^{4} x \mathcal{L}_{e f f}\right],
$$

where $\mathcal{L}_{\text {eff }}$ is the effective Lagrangian of the system and can be written as,

$$
\begin{aligned}
& \mathcal{L}_{\text {eff }}=\left|\partial_{I} \pi\right|^{2}-\left(m^{2}+\frac{\lambda}{6} \phi^{2}\right)|\pi|^{2}+\frac{1}{2}\left(\partial \pi_{0}\right)^{2}+\frac{1}{2}(\partial \sigma)^{2} \\
& -\frac{1}{2}\left(m^{2}+\frac{\lambda}{6} \phi^{2}\right) \pi_{0}^{2}-\frac{1}{2}\left(m^{2}+\frac{\lambda}{2} \phi^{2}\right) \sigma^{2}-\frac{1}{2} m^{2} \phi^{2}-\frac{\lambda}{24} \phi^{4} \\
& -\frac{\lambda}{24}\left[\sigma^{4}+4|\pi|^{4}+\pi_{0}^{4}+4 \sigma^{2}|\pi|^{2}+2 \sigma^{2} \pi_{0}^{2}+4|\pi|^{2} \pi_{0}^{2}\right]
\end{aligned}
$$

with $\left|\partial_{I} \pi\right|^{2}=\left(\partial_{\mu}+i \mu \delta_{\mu 0}\right) \pi_{+}\left(\partial^{\mu}-i \mu \delta^{\mu 0}\right) \pi_{-}$and $|\pi|^{2}=\pi_{+} \pi_{-}$. It should be noted that the chemical potential $\mu$ has entered the lagrangian in $\left|\partial_{I} \pi\right|^{2}$. The following calculations and discussions will be based on this effective Lagrangian.

\section{Gap equations and thermodynamic potential}

Within the imaginary time formalism of finite temperature field theory, we have

$$
\int \frac{d^{4} k}{(2 \pi)^{4}} f(k) \rightarrow \frac{1}{\beta} \sum_{n} \int \frac{d^{3} \mathbf{k}}{(2 \pi)^{3}} f\left(i \omega_{n}, \mathbf{k}\right) \equiv \int_{\beta} f\left(i \omega_{n}, \mathbf{k}\right),
$$

where $\beta$ is the inverse temperature, $\beta=1 / T$; the integration over the time component $k_{0}$ has been replaced by a summation over discrete frequencies. For boson there are $\omega_{n}=2 \pi n T$ and $n=0, \pm 1, \pm 2, \cdots$. For the sake of simplicity in what follows, a shorthand notation is used to denote the integration and the summation.

According to the Lagrangian (8), the tree level inverse propagators of $\sigma, \pi_{0}$ and $\pi_{ \pm}$ can be written respectively as

$$
D_{\sigma}^{-1}=\omega_{n}^{2}+\mathbf{k}^{2}+m^{2}+\frac{\lambda}{2} \phi^{2},
$$


Bose-Einstein condensation and chiral phase transition in linear sigma model

$$
\begin{aligned}
& D_{0}^{-1}=\omega_{n}^{2}+\mathbf{k}^{2}+m^{2}+\frac{\lambda}{6} \phi^{2}, \\
& D^{-1}=\left(\omega_{n}+i \mu\right)^{2}+\mathbf{k}^{2}+m^{2}+\frac{\lambda}{6} \phi^{2} .
\end{aligned}
$$

The equation (12) represents the inverse propagator of $\pi_{+}$and $\pi_{-}$. As the summation in equation (9) is symmetric over $n$ from $-\infty$ to $+\infty, \omega_{n}+i \mu$ and $\omega_{n}-i \mu$ are equivalent in describing the propagators of $\pi_{+}$and $\pi_{-}$. Here we just choose $\omega_{n}+i \mu$ to describe both $\pi_{+}$and $\pi_{-}$, and write the two propagators in the same form as in equation (12). According to [10], we can write down the corresponding effective potential at finite temperature as

$$
\begin{gathered}
V(\phi, G)=\frac{1}{2} m^{2} \phi^{2}+\frac{\lambda}{24} \phi^{4}+\frac{1}{2} \int_{\beta} \ln G_{\sigma}^{-1}+\frac{1}{2} \int_{\beta}\left[D_{\sigma}^{-1} G_{\sigma}-1\right]+\frac{1}{2} \int_{\beta} \ln G_{0}^{-1} \\
+\frac{1}{2} \int_{\beta}\left[D_{0}^{-1} G_{0}-1\right]+\int_{\beta} \ln G^{-1}+\int_{\beta}\left[D^{-1} G-1\right]+V_{2}(\phi, G),
\end{gathered}
$$

where $G_{\sigma}, G_{0}$ and $G$ are the full propagators of $\sigma, \pi_{0}$ and $\pi_{ \pm}$respectively. They are determined by the stationary condition (1). The chemical potential is included in the full propagator. $V_{2}(\phi, G)$ represents the infinite sum of the two particle irreducible vacuum graphs. However, in CJT formalism at the Hartree approximation we need only to calculate the "double bubble" diagrams with four-point vertex and treat each loop line as the full propagator [10, 15]. Therefore, $V_{2}$ can be written as

$$
\begin{aligned}
V_{2}(\phi, G) & =\frac{\lambda}{8}\left[\int_{\beta} G_{\sigma}\right]^{2}+\frac{\lambda}{3}\left[\int_{\beta} G\right]^{2}+\frac{\lambda}{8}\left[\int_{\beta} G_{0}\right]^{2} \\
& +\frac{\lambda}{6} \int_{\beta} G_{\sigma} \int_{\beta} G+\frac{\lambda}{12} \int_{\beta} G_{\sigma} \int_{\beta} G_{0}+\frac{\lambda}{6} \int_{\beta} G \int_{\beta} G_{0} .
\end{aligned}
$$

By minimizing the effective potential $V(\phi, G)$ with respect to the full propagators, we get the following set of nonlinear gap equations,

$$
\begin{aligned}
& G_{\sigma}^{-1}=D_{\sigma}^{-1}+\frac{\lambda}{2} \int_{\beta} G_{\sigma}+\frac{\lambda}{3} \int_{\beta} G+\frac{\lambda}{6} \int_{\beta} G_{0}, \\
& G_{0}^{-1}=D_{0}^{-1}+\frac{\lambda}{2} \int_{\beta} G_{0}+\frac{\lambda}{6} \int_{\beta} G_{\sigma}+\frac{\lambda}{3} \int_{\beta} G, \\
& G^{-1}=D^{-1}+\frac{2 \lambda}{3} \int_{\beta} G+\frac{\lambda}{6} \int_{\beta} G_{\sigma}+\frac{\lambda}{6} \int_{\beta} G_{0} .
\end{aligned}
$$

Furthermore we take the similar ansatz for the full propagators as in [10],

$$
\begin{aligned}
& G_{\sigma}^{-1}=\omega_{n}^{2}+\mathbf{k}^{2}+M_{\sigma}^{2}, \\
& G_{0}^{-1}=\omega_{n}^{2}+\mathbf{k}^{2}+M_{0}^{2}, \\
& G^{-1}=\left(\omega_{n}+i \mu\right)^{2}+\mathbf{k}^{2}+M^{2},
\end{aligned}
$$

where the effective masses $M_{\sigma}, M_{0}$ and $M$ have been introduced for $\sigma, \pi_{0}$ and $\pi_{ \pm}$ respectively. Substituting them into the above gap equations, we obtain a set of effective mass gap equations,

$$
M_{\sigma}^{2}=m^{2}+\frac{\lambda}{2} \phi^{2}+\frac{\lambda}{2} \int_{\beta} G_{\sigma}+\frac{\lambda}{3} \int_{\beta} G+\frac{\lambda}{6} \int_{\beta} G_{0},
$$


Bose-Einstein condensation and chiral phase transition in linear sigma model

$$
\begin{aligned}
& M_{0}^{2}=m^{2}+\frac{\lambda}{6} \phi^{2}+\frac{\lambda}{2} \int_{\beta} G_{0}+\frac{\lambda}{6} \int_{\beta} G_{\sigma}+\frac{\lambda}{3} \int_{\beta} G, \\
& M^{2}=m^{2}+\frac{\lambda}{6} \phi^{2}+\frac{2 \lambda}{3} \int_{\beta} G+\frac{\lambda}{6} \int_{\beta} G_{\sigma}+\frac{\lambda}{6} \int_{\beta} G_{0} .
\end{aligned}
$$

Accordingly the effective potential can be written as

$$
\begin{aligned}
V(\phi, M) & =\frac{1}{2} m^{2} \phi^{2}+\frac{\lambda}{24} \phi^{4}+\frac{1}{2} \int_{\beta} \ln G_{\sigma}^{-1} \\
& -\frac{1}{2} \int_{\beta}\left(M_{\sigma}^{2}-m^{2}-\frac{\lambda}{2} \phi^{2}\right) G_{\sigma}+\frac{1}{2} \int_{\beta} \ln G_{0}^{-1} \\
& -\frac{1}{2} \int_{\beta}\left(M_{0}^{2}-m^{2}-\frac{\lambda}{6} \phi^{2}\right) G_{0}+\int_{\beta} \ln G^{-1} \\
& -\int_{\beta}\left(M^{2}-m^{2}-\frac{\lambda}{6} \phi^{2}\right) G+V_{2}(\phi, M) .
\end{aligned}
$$

By minimizing the potential with respect to the order parameter $\phi$, we obtain one more equation,

$$
m^{2} \phi+\frac{\lambda}{6} \phi^{3}+\frac{\lambda \phi}{2} \int_{\beta} G_{\sigma}+\frac{\lambda \phi}{6} \int_{\beta} G_{0}+\frac{\lambda \phi}{3} \int_{\beta} G=0 .
$$

From equations (21) - (23) and (25), the effective masses and order parameter can be solved self-consistently at given temperature and chemical potential. There are some discussions concerning the renormalization on the CJT formalism in different models [16, 18, 19]. Recent investigation about the renormalization of the $\mathrm{O}(\mathrm{N})$ linear sigma model has been addressed in [11. In our discussion, the divergent parts of the integrals are neglected as is done in [10]. And we take the finite parts of the integrals as follows:

$$
\begin{aligned}
& \int_{\beta} \ln G^{-1}=\int_{\beta} \ln \left[\left(\omega_{n}+i \mu\right)^{2}+\mathbf{k}^{2}+M^{2}\right] \\
= & \frac{1}{\beta} \int \frac{d^{3} \mathbf{k}}{(2 \pi)^{3}}\left[\ln \left(1-e^{-\beta(\omega+\mu)}\right)+\ln \left(1-e^{-\beta(\omega-\mu)}\right)\right], \\
& \int_{\beta} G=\int_{\beta} \frac{1}{\left(\omega_{n}+i \mu\right)^{2}+\mathbf{k}^{2}+M^{2}} \\
= & \int \frac{d^{3} \mathbf{k}}{(2 \pi)^{3}} \frac{1}{2 \omega}\left[\frac{1}{e^{\beta(\omega+\mu)}-1}+\frac{1}{e^{\beta(\omega-\mu)}-1}\right],
\end{aligned}
$$

where $\omega=\sqrt{\mathbf{k}^{2}+M^{2}}$. The integration over $G_{0}$ and $G_{\sigma}$ will be dealt with in the same way. With the chiral limit $(\varepsilon=0)$, the corresponding coupling constant and the negative mass parameter can be taken as $\lambda \approx 125$ and $-m^{2} \approx 1.8 \times 10^{5} \mathrm{MeV}^{2}$.

In the discussion of the thermodynamic system, the effective potential $V$ is equivalent to the thermodynamic potential $\Omega$. Thus we get

$$
\begin{aligned}
\Omega & =\frac{1}{2} m^{2} \phi^{2}+\frac{\lambda}{24} \phi^{4}+T \int \frac{d^{3} \mathbf{k}}{(2 \pi)^{3}} \ln \left(1-e^{-\beta \omega_{\sigma}}\right) \\
& -\frac{1}{2}\left(M_{\sigma}^{2}-m^{2}-\frac{\lambda}{2} \phi^{2}\right) \int \frac{d^{3} \mathbf{k}}{(2 \pi)^{3}} \frac{1}{\omega_{\sigma}} \frac{1}{e^{\beta \omega_{\sigma}}-1}
\end{aligned}
$$




$$
\begin{aligned}
& +T \int \frac{d^{3} \mathbf{k}}{(2 \pi)^{3}} \ln \left(1-e^{-\beta \omega_{0}}\right)-\frac{1}{2}\left(M_{0}^{2}-m^{2}-\frac{\lambda}{6} \phi^{2}\right) \int \frac{d^{3} \mathbf{k}}{(2 \pi)^{3}} \frac{1}{\omega_{0}} \frac{1}{e^{\beta \omega_{0}}-1} \\
& +T \int \frac{d^{3} \mathbf{k}}{(2 \pi)^{3}}\left[\ln \left(1-e^{-\beta(\omega+\mu)}\right)+\ln \left(1-e^{-\beta(\omega-\mu)}\right)\right] \\
& -\left(M^{2}-m^{2}-\frac{\lambda}{6} \phi^{2}\right) \int \frac{d^{3} \mathbf{k}}{(2 \pi)^{3}} \frac{1}{2 \omega}\left[\frac{1}{e^{\beta(\omega+\mu)}-1}+\frac{1}{e^{\beta(\omega-\mu)}-1}\right]+\Omega_{2},
\end{aligned}
$$

where $\Omega_{2}=V_{2}, \omega_{\sigma}=\sqrt{\mathbf{k}^{2}+M_{\sigma}^{2}}, \omega_{0}=\sqrt{\mathbf{k}^{2}+M_{0}^{2}}$ and $\omega=\sqrt{\mathbf{k}^{2}+M^{2}}$. According to the relation

$$
\rho=-\frac{\partial \Omega}{\partial \mu}
$$

and equation (23), we can get the net charge density

$$
\rho=\int \frac{d^{3} \mathbf{k}}{(2 \pi)^{3}}\left[\frac{1}{e^{\beta(\omega-\mu)}-1}-\frac{1}{e^{\beta(\omega+\mu)}-1}\right] .
$$

This expression of density seems very similar to that of ideal gas, but actually they are different. Because here the effective mass $M$ in $\omega$ is a function of temperature and will be determined self-consistently by the gap equations.

\section{BEC and chiral phase transition}

Now we are in a position to discuss BEC and the chiral phase transition. We will study BEC first. It is known that when $\mu=M$, BEC occurs. The equation (30) should be written as

$$
\begin{aligned}
& \rho=\rho_{0}+\rho^{*}(\beta, \mu=M), \\
& \rho^{*}(\beta, \mu=M)=\int \frac{d^{3} \mathbf{k}}{(2 \pi)^{3}}\left[\frac{1}{e^{\beta(\omega-M)}-1}-\frac{1}{e^{\beta(\omega+M)}-1}\right],
\end{aligned}
$$

where $\rho_{0}$ represents the charge density of the zero-momentum state [17, 20]. Let us determine the point at which $\mu=M$ is reached. From equation (30), when $\rho$ is fixed, by solving the gap equations (21) - (23) and equation (25), we find both $\mu$ and $M$ are functions of $T$. It can be plotted in figure 1 . We can see that with $T$ decreasing, $M$ decreases first and then increases, while $\mu$ keeps increasing quickly and approaches $M$. At certain temperature, $\mu$ catches up with $M$ and BEC happens. From equation (31), we know the critical temperature $T_{c}$ is determined implicitly by the equation

$$
\rho=\rho^{*}\left(\beta_{c}, \mu=M\right) .
$$

When $T \leq T_{c}$, the system goes into BEC phase. The equation (31) will be solved together with the gap equations with $\rho$ fixed. We find $\mu$ and $M$ are still functions of $T$ and $\mu(T)=M(T)$. They both decrease with temperature decreasing, which is indicated in figure 1.

From equation (31) which is solved together with the gap equations at fixed $\rho$, we can get $\rho_{0}$ as a function of $T$. This is shown in figure 2. It is clear that when $\rho$ is fixed, at $T>T_{c}, \rho_{0}=0$; at $T \leq T_{c}, \rho_{0}$ starts to increase quickly with temperature decreasing, 


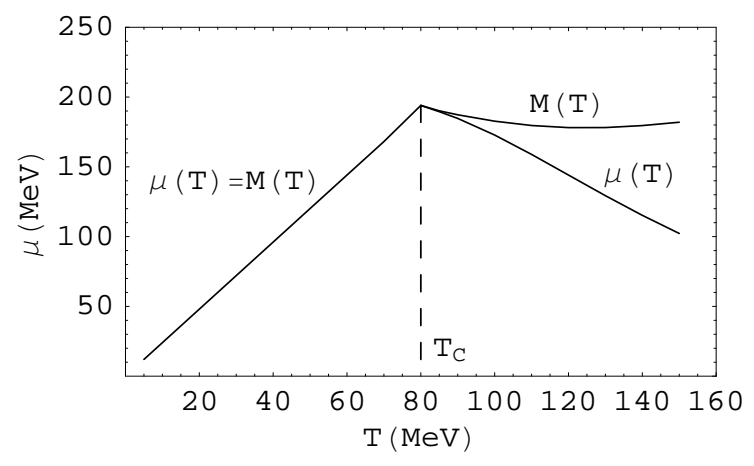

Figure 1. $\mu$ and $M$ as functions of $T$ at the fixed total charge density $\rho=0.06 \mathrm{fm}^{-3}$. $\mathrm{BEC}$ happens at $T_{c}=80 \mathrm{MeV}$.

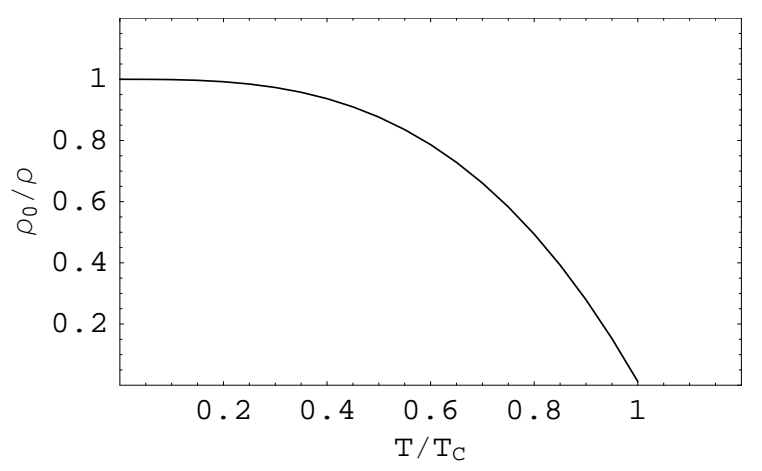

Figure 2. The ratio $\rho_{0} / \rho$ as a function of the ratio $T / T_{c}$ at $\rho=0.06 \mathrm{fm}^{-3}$ and $T_{c}=80 \mathrm{MeV}$.

which means large amount of the charged pions will reside to the zero momentum state and form BEC.

For different fixed total density $\rho$, there will be different values of critical temperature $T_{c}$ and chemical potential $\mu\left(T_{c}\right)$, so one can study the $\mu-T$ phase diagram of BEC. In solving the self-consistent equations, we observe that $T_{c}$ and $\mu\left(T_{c}\right)$ depend on the order parameter $\phi$. In low temperature regime, the order parameter $\phi$ is nonzero and determined by equation (25). When it is solved together with the gap equations (21) - (23) and equation (32), the critical temperature of BEC at a given density can be determined. The $\mu-T$ phase diagram for BEC can be given accordingly as shown in figure 3. The dividing line which separates BEC and the normal phase at $\phi \neq 0$ is curve AC. At $T>T_{C}$ (the temperature at the point $\mathrm{C}$ ), there is no solution with $\phi \neq 0$ for the onset of BEC. Because in the high temperature regime the chiral symmetry is restored and $\phi=0$. At $\phi=0$ the phases dividing line is curve DF as shown in figure 3. We can see that BEC happens in chiral symmetry broken state and restored state at low temperature and high temperature respectively.

In the regime of $T_{B}<T<T_{C}$, it seems that BEC can happen at both $\phi \neq 0$ and $\phi=0$. However, if we calculate thermodynamic potential from equation (28), we find 
under the same temperature the thermodynamic potential of $\phi \neq 0$ has lower values than that of $\phi=0$, which means at $T_{B}<T<T_{C}$, BEC would happen along the curve $\mathrm{BC}$. Then the final $\mu-T$ phase diagram of $\mathrm{BEC}$ is determined and shown in figure 4 . In low temperature regime $\mathrm{BEC}$ phase and the normal phase are divided by curve $\mathrm{AC}$, while in high temperature regime, the phases are divided by curve EF. The whole phase plane has been divided into two phase areas. The area above the curve belongs to the BEC phase, while the area below the curve represents the normal phase.

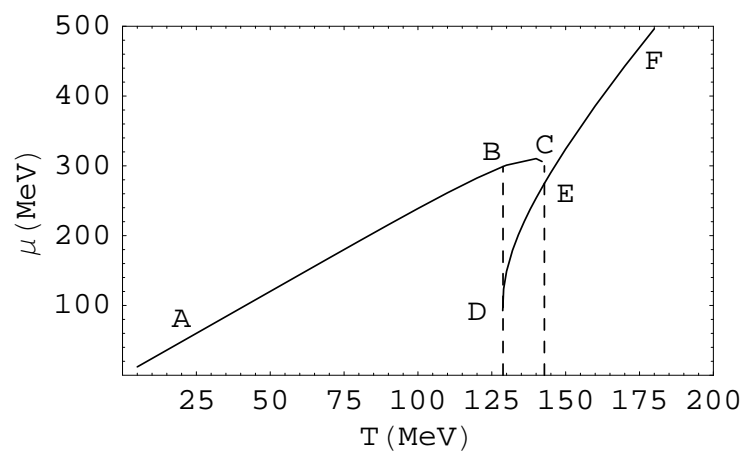

Figure 3. The phase diagram of $\mu$ versus $T$ for BEC. (AC and DF are the dividing lines which separate BEC and normal phases in low temperature regime and high temperature regime respectively.)

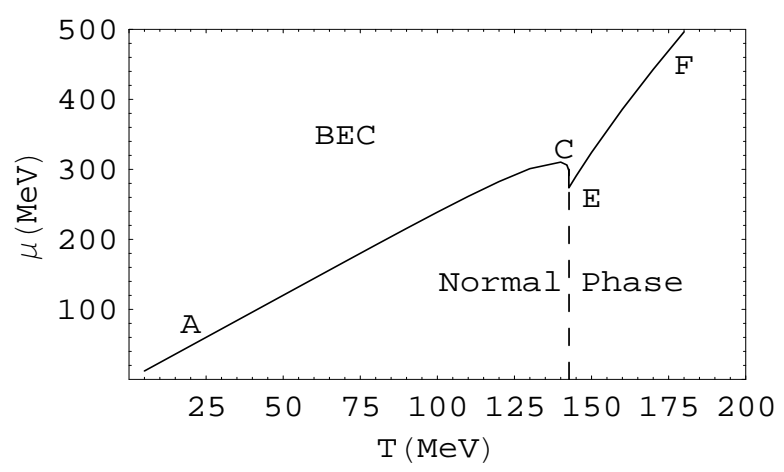

Figure 4. The $\mu-T$ phase diagram for BEC with no ambiguity.

Now we will discuss the chiral phase transition in this system. By using the linear sigma model, there are many discussions on the chiral phase transition at finite temperature [10, 11, 12]. As we have considered finite pion density as well as finite temperature, the calculation becomes more involved. However, the chiral phase transition is still characterized by the order parameter $\phi$. In our discussion, $\phi$ is not only temperature dependent but also chemical potential dependent. In [10], the chiral phase transition has been discussed at finite temperature with the linear sigma model. By using CJT resummation scheme in Hartree approximation, the transition is found to be first order in the chiral limit. If we take $\mu=0$, we can reproduce their result. 


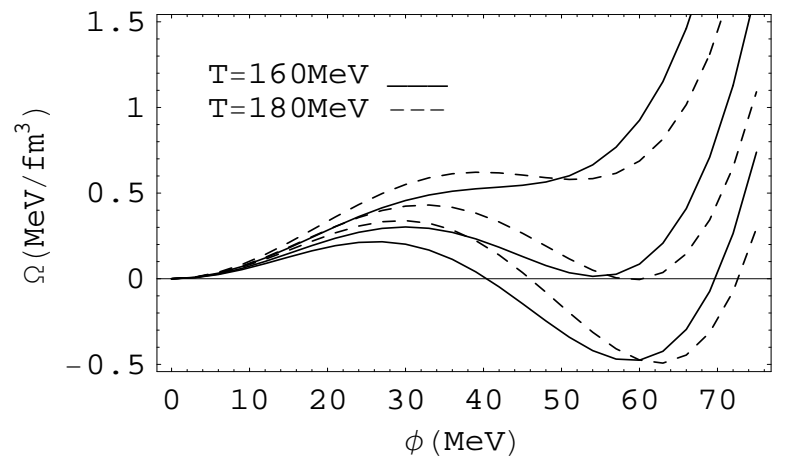

Figure 5. $\Omega$ as functions of $\phi$. Solid lines stand for $T=160 \mathrm{MeV}$, and the chemical potentials for different lines are $260 \mathrm{MeV}, 237 \mathrm{MeV}$ and $220 \mathrm{MeV}$ respectively (from top to bottom). Dashed lines stand for $T=180 \mathrm{MeV}$, and the chemical potentials are $140 \mathrm{MeV}, 100 \mathrm{MeV}$ and $60 \mathrm{MeV}$ respectively (also from top to bottom).

In the case of $\mu \neq 0$, we find the chiral phase transition is still first order. This can be seen if we take a look at $\Omega$ as a function of $\phi$. As the gap equations (21) - (23) can be solved at certain $T$ and $\mu$, from equation (28), the thermodynamical potential $\Omega$ can be determined. The $\phi$ dependence of $\Omega$ can be plotted as shown in figure 5 . In the transition, it can be seen that there are two minima of $\Omega$ which correspond to the stable and meta-stable states at certain $T$ and $\mu$. If $T$ is fixed, by increasing $\mu$, the lower minimum of $\phi \neq 0$, which stays in a stable state, will be lifted up. When it becomes higher than the $\phi=0$ minimum, it changes to a meta-stable state from the stable state. In other words, the $\phi=0$ minimum becomes a stable state which means chiral symmetry is restored. It is clear that the chiral phase transition is first order. The transition is usually defined at the point where the two minima become degenerate. In figure 5, when $T=160 \mathrm{MeV}$ and $T=180 \mathrm{MeV}$, we can see that the transitions happen at $\mu=237 \mathrm{MeV}$ and $\mu=100 \mathrm{MeV}$ respectively. According to this way, the $\mu-T$ phase diagram for the chiral phase transition can be plotted as shown in figure 6 .

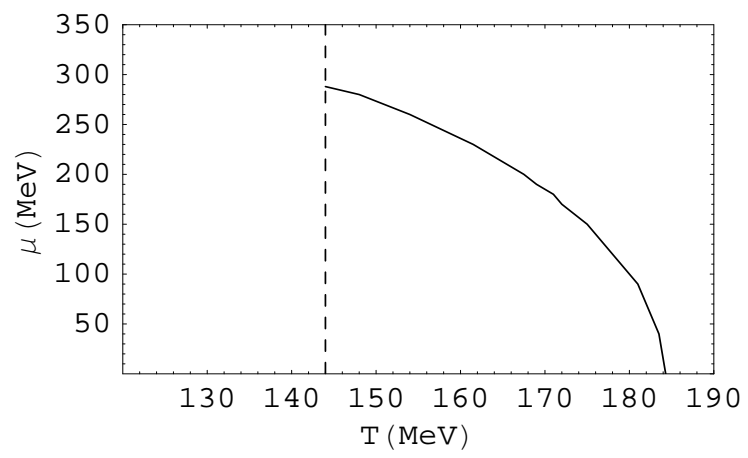

Figure 6. The phase diagram of $\mu$ versus $T$ for the chiral phase transition. When $T>144 \mathrm{MeV}$, the area above the curve is the restored phase; the area below the curve is the broken phase. 
From figure 6, we can see that the transition temperature is $184 \mathrm{MeV}$ for $\mu=0$, which was obtained in 10 . Here when $\mu \neq 0$, the transition temperature will decrease with the chemical potential increasing. At certain temperature $(T \approx 144 \mathrm{MeV})$, the phase curve of the chiral phase transition will terminate, because at this time $\mu=M$ and BEC will happen. As a result the chiral phase transition can not take place further at lower temperatures. When $T>144 \mathrm{MeV}$, the area above the curve is the chiral symmetry restored phase. However, the phase diagram is not complete, because we do not know what the phase is when $T<144 \mathrm{MeV}$. Now let us recall the $\mu-T$ phase diagram of BEC (figure 4). If we combine the two phase diagrams (figure 4 and figure 6) into one figure, we can see the different phase areas clearly. This is shown in figure 7. When the chiral phase transition terminates, it means that the system will go into BEC phase. Furthermore, the normal phase area in figure 4 is divided into the chiral symmetry broken phase and the chiral symmetry restored phase. When the system is in a chiral symmetry broken phase, if we decrease temperature with density fixed, BEC will occur at ceratin temperature; if we increase temperature, the system may go into a chiral symmetry restored phase. In the restored phase, at relatively low densities, if we decrease temperature, the chiral phase transition may take place; at relatively high densities, if we decrease temperature with density fixed, BEC will take place.

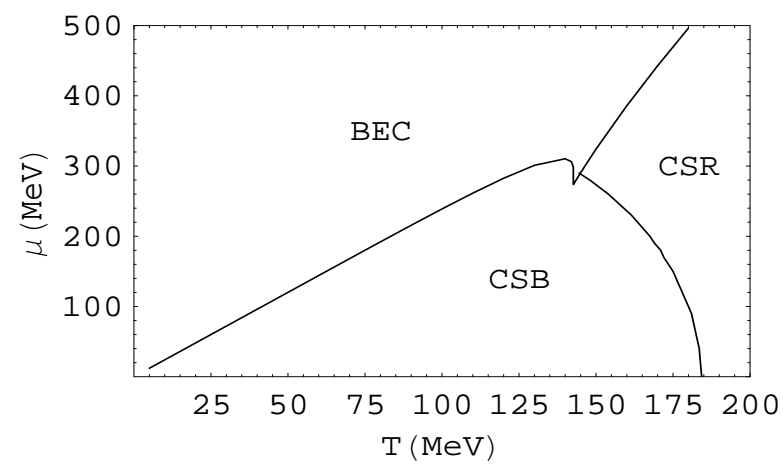

Figure 7. The combined phase diagram of $\mu$ versus $T$ for BEC and the chiral phase transition. The CSB and CSR stand for chiral symmetry broken phase and chiral symmetry restored phase respectively.

We noted that in 8], BEC and the spontaneous symmetry breaking in interacting Bose system have been discussed with the $\mathrm{O}(\mathrm{N})$ model. In their discussion, a chemical potential $\mu$ associated with $\mathrm{O}(2)$ symmetry is introduced when $\mathrm{N}$ is even. There are $N / 2$ such chemical potentials for $N$ scalars. For only one chemical potential and the large-N limit, their result shows that the transition to broken symmetry phase is just the onset of $\mathrm{BEC}$ in the the ultra-relativistic regime. This is because the scalar field associated with $\mu$ acquires a nonzero vacuum expectation value at the point that $\mu=M$ is reached when temperature is lowered. Thus the symmetry, which is actually the $\mathrm{O}(2)$ symmetry, is broken at this time. In our discussion, we have studied the chiral symmetry restoration and $\mathrm{BEC}$ in the $\mathrm{O}(4)$ linear sigma model. In this model, the four scalar fields have real 
physical meaning, which are three pion fields and one sigma field. The chiral symmetry breaking in the vacuum is chosen along the $\sigma$ direction. The chiral phase transition is characterized by the order parameter $\phi$. The chemical potential here is associated with the pion fields. The BEC means the BEC of the charged pions. The relation between BEC and the chiral phase transition can be illustrated by the $\mu-T$ phase diagram as indicated in figure 7 .

\section{Conclusion}

In summary, we have discussed Bose-Einstein condensation and the chiral phase transition for the linear sigma model in the chiral limit. The effective potential of the linear sigma model is calculated within the CJT resummation scheme in the Hartree approximation at finite temperature and finite pion density. By solving the self-consistent equations we have discussed BEC in the interacting pion system. The $\mu-T$ phase diagram of BEC is presented. At relatively low temperature, BEC happens in chiral symmetry broken state, while at relatively high temperature, BEC happens in chiral symmetry restored state. The phase plane has been divided into the BEC phase and the normal phase. Then we have discussed the chiral phase transition and also plotted the $\mu-T$ phase digram of the chiral phase transition. By comparing it

with the BEC phase diagram, we find the chiral phase transition happens in the normal phase and further divided the normal phase into the chiral symmetry broken phase and restored phase. So the whole phase plane has been divided into three phase areas: the BEC phase area, the chiral symmetry broken phase area and the chiral symmetry restored phase area. BEC can happen either from the chiral symmetry broken phase or from the chiral symmetry restored phase. When the system goes into BEC phase, there is no chance for the onset of the chiral phase transition.

\section{Acknowledgments}

We would like to thank Professor Ji-Sheng Chen and Professor De-Fu Hou for their help. This work was supported in part by the National Natural Science Foundation of China with No. 90303007.

\section{References}

[1] Greiner C, Gong C and Müller B 1993 Phys. Lett. B 316226

[2] Florkowski W and Abu-Samreh M 1996 Z. Phys. C 70133

[3] Ayala A and Smerzi A 1997 Phys. Lett. B 40520

[4] Bialas A and Zalewski K 1999 Phys. Rev. D 59097502

[5] Zalewski K 2000 Nucl. Phys. Proc. Suppl. 8636

[6] Karsch F 2002 Lect.Notes Phys. 583209

[7] Kapusta J 1981 Phys. Rev. D 24426

[8] Haber H and Weldon H 1982 Phys. Rev. D 25502

[9] Dobado A and Pelaez J 1999 Phys. Rev. D 59034004 
[10] Petropoulos Nicholas 1999 J. Phys. G 252225

[11] Lenaghan J and Rischke D 2000 J. Phys. G 26431

[12] Scavenius O et al 2001 Phys. Rev. C 64045202

[13] Ayala A, Amore P and Aranda A 2002 Phys. Rev. C 66045205

[14] Loewe M and Villavicencio C 2003 Phys. Rev. D 67074034

[15] Cornwall J, Jackiw R and Tomboulis E 1974 Phys. Rev. D 102428

[16] Amelino-Camelia G and Pi So-Young 1993 Phys. Rev. D 472356

[17] Kapusta J 1989 Finite-Temperature Field Theory (Cambridge Univ. Press)

[18] Amelino-Camelia G 1997 Phys. Lett. B 407268

[19] Amelino-Camelia G 1996 Nucl. Phys. B 476255

[20] Pathria R 1972 Statistical Mechanics (Pergamon Press) 